\title{
El "museo de la psicología" como estrategia para la construcción de la historia oral de la psicología
}

\section{The "Museum of Psychology" as a Strategy for the} Construction of the Oral History of Psychology

Recibido: 26 de enero de 2014 | Revisado: 26 de febrero de 2014 | Aceptado: 17 de juliio de 2014

\author{
Hernán Camilo Pulido Martínez* \\ LUZ MERY CARVAJAL MARÍN ** \\ LiNA María Plata CASTILlO *** \\ VERÓNICA RIVERA SERNA *****
}

Pontificia Universidad Javeriana, Bogotá, Colombia

doi:10.11144/Javeriana.upsy13-5.mpec

Para citar este artículo: Pulido, H. C., Carvajal, L. M., Plata, L. M., \& Rivera, V. (2014). El "museo de la psicología" como estrategia para la construcción de la historia oral de la psicología. Universitas Psychologica, 13(5), 1905-1917. http://dx.doi.org/10.11144/ Javeriana.upsy13-5.mpec

\footnotetext{
PhD.Correo electrónico: cpulido@Javeriana.edu. $\mathrm{CO}$

** Ms. Sc. Correo electrónico: lmcarvaj@javeriana. edu.co

****Correo electrónico: linaplata@hotmail.com

***** Correo electrónico: verorivera89@gmail.com
}

\begin{abstract}
RESUMEN
En este artículo, se presenta la construcción de un "museo de la psicología" como estrategia para la elaboración de la historia oral de la disciplina. En primer lugar, el debate entre psicología e historia constituye el marco para considerar el puesto que pueden ocupar los relatos de la experiencia de la práctica psicológica, en relación con la constitución de la historia de esta disciplina. A continuación, se describen los elementos fundamentales de la estrategia llamada "museo de la psicología", diseñada para elicitar historias orales. Para finalizar, a manera de ilustración, se consideran ventajas y limitaciones que emergen de la aplicación de la estrategia.

Palabras clave

historia de la psicología; historia oral; museo; experiencia; psicologización

\section{A B S T R A C T}

In this article the construction of a "museum of psychology" is presented as a strategy for the elaboration of the oral history of the discipline. First of all, the debate between history and psychology constitutes the framework to consider the place of psychological practices as regards the construction of the history of psychology. Then, the fundamental elements of the strategy called "the museum of psychology" are introduced. Finally, as an illustration, the advantages and the limitations of the strategy are presented. Keywords

history of psychology; oral history; museum; experience; psychologization
\end{abstract}




\section{Introducción}

Desde hace ya varias décadas, algunos investigadores en el campo de la historia de la disciplina se han dado a la tarea de recolectar, en archivos especializados, un acervo de historias orales de psicólogos, con el objeto de preservar las memorias de la experiencia como insumo básico para la práctica de la investigación. Por ejemplo, fácilmente se encuentran referencias en el internet al archivo de historia oral vinculado con la British Psychological Association (http://hopc.bps.org.uk/collarch/jkaa/ ohp.cfm), cuyas primeras grabaciones datan del año 1956. Archivo pionero que se ha ocupado de la recolección de información referida a aspectos académicos y profesionales del periodo formativo de la psicología que acaecieron en el Reino Unido. También se está llevando a cabo la construcción del archivo de historias orales en universidad de York (http://ists.news.yorku.ca/projects/psychologys-feminist-voices/), cuyo énfasis se ha puesto en la recolección de narraciones de mujeres psicólogas, tradicionalmente olvidadas en las versiones oficiales de la disciplina, con el fin de proporcionar insumos para la investigación histórica de corte feminista. El archivo de historias orales de los psicólogos del desarrollo, patrocinado por la Society for Research in Child Development (http://www.srcd.org/about-us/ oral-history-project), y el archivo de historias orales de la psicología de la Universidad de Melbourne (http://gallery.its.unimelb.edu.au/imu/imu.php?requ est=multimedia\&irn=4938), son empresas académicas que están llevando a cabo una recopilación de la historia de la psicología desde los mismos protagonistas.

La investigación histórica con base en los relatos directos de los psicólogos representa ventajas a la hora de dar respuesta a preguntas relacionadas con la práctica psicológica. Registros de las interacciones cotidianas en las instituciones en donde se han desempeñado los psicólogos no son fáciles de encontrar. Por tanto, no se tiene mayor información sobre cómo los objetos psicológicos, en su mayoría producidos en las sociedades del Atlántico Norte, son, por ejemplo, replicados, adaptados e hibridados en otros contextos sociales. Quiere esto decir que las dificultades cotidianas enfrentadas para llegar a aplicar la psicología, los problemas confrontados con las técnicas psicológicas, los actores involucrados, los sentimientos que se suscitaron, los debates llevados a cabo, así como, las apropiaciones particulares hechas localmente, no se encuentran con mucha frecuencia consignadas en documentos escritos. La historia oral resulta entonces una herramienta útil para conocer los usos cotidianos que se han hecho de la psicología, en los muy diversos ámbitos a donde esta ha llegado.

A este respecto de los usos, la historia oral permite hacer un desplazamiento en términos de la mirada al pasado que se construye. No es, por supuesto, el género de historia de la psicología referido a las grandes ideas y personajes de la disciplina, el cual parece destinado a legitimar este conocimiento y a socializar a los nuevos estudiantes (Klappenbach, 2006; Vezzetti, 1996). Tampoco se trata de la "historia crítica" proveniente de los marcos conceptuales formulados para examinar a la psicología como cuerpo moderno de conocimientos (Jaraba-Barros \& Mora-Gamez, 2010; Rose, 1996). Se podría decir que, en términos de la construcción de la historia de la psicología, la historia oral representa más bien una forma "austera" y complementaria de mirar al pasado (Fraser, 1993; Portelli, 2003; Thomson, 2007), tanto por centrarse en lo local y cotidiano de la psicología como porque sus protagonistas son aquellos psicólogos que, en ocasiones, no sin un dejo de desprecio, han sido llamados "grises", por su falta de rutilancia dentro de los cánones académicos. Estos psicólogos no determinaron el curso que debía llevar la disciplina, no obstante, desempeñan un papel central cuando se tienen en cuenta las geopolíticas del conocimiento. Gracias a ellos, "verdaderamente", la psicología se ha tornado en un conocimiento de carácter universal. Sin tener una intención explícita, en gran parte por su trabajo como diseminadores, la psicología ha logrado colonizar ámbitos sociales de muy diverso orden, llevando a hacer propuestas comunes acerca de cómo son los seres humanos, cómo deben comportarse y cómo deben llevarse a cabo ciertas prácticas para "conducir su conducta" (Foucault, 1979). 
El "MUSEO de la psicología” como estrategia para la

CONSTRUCCIÓN DE LA HISTORIA ORAL DE LA PSICOLOGÍA

Sin embargo, se presenta una carencia central: las prácticas de estos profesionales permanecen olvidadas en las historias de la psicología. Paradójica situación, por una parte su práctica es indispensable para entender el lugar que tiene y las operaciones que cumple este conocimiento. Por otra parte, no se le ha dado el interés que debe suscitar su actuar cotidiano, el cual lleva a la instauración, en muy diversos ámbitos, de aquella psicología "mundana" de la que se dice es uno de los vehículos conducentes a la psicologización de las sociedades (Illouz, 2010; Jansz \& Van Drunen, 2003; Pfister \& Schnog, 1997; Triantafillou \& Moreira, 2005; Ward 2002).

En este artículo, en el marco del debate entre historia e historia oral, se presenta la construcción de un "museo de la psicología" como una estrategia metodológica, diseñada para elicitar historias de la práctica psicológica. Esta estrategia hace eco, entre otros, de procesos investigativos más horizontales en donde investigador e investigado se alían para dirigirse a la construcción de versiones sobre lo ocurrido, en términos de los usos, las emociones, las aplicaciones e hibridaciones de la psicología. A manera de ilustración, se incluye un fragmento resultado de la aplicación del "museo de la psicología" a un grupo de profesionales de esta disciplina, con el propósito de señalar las posibilidades y limitaciones que esta puede tener para construir narraciones del pasado de la práctica psicológica, desde la perspectiva de sus propios protagonistas.

\section{Historia y psicología}

El interés creciente por la historia de la psicología ha llevado a los investigadores a reflexionar sobre las distintas formas en que se ha configurado la relación entre estas dos disciplinas, así como a considerar lo que podría ser el futuro del campo (Danziger, 1994; Lovett, 2006; Samelson, 1999). Significativamente, la discusión acerca de los vínculos existentes entre la psicología y la historia permanece sin extenderse a las distintas áreas de la disciplina. Sin embargo, este debate ha llevado a cambios en la concepción de lo que es la psicología, de su lugar social, de las operaciones que cumple y, por supuesto, a reconsiderar las relaciones mismas entre historia y psicología (Castel, Castel, \& Lowell, 1980; Danziger, 2001; Eghigian, Killen, \& Luenberger, 2007; Gillespie, 1993; Herman, 1995; Rose, 1996, 1999). Las preguntas centrales para el debate, lejos de habérseles dado una respuesta definitiva, siguen a la orden día. Según GI $\square$ veanu y Yamamoto (2012), por ejemplo, preguntas tales como, ien qué puede consistir un puente entre la psicología y la historia?, icómo se puede construir dicho puente? y ipor qué razones hay que constituir esta relación? son las guías de aquellos interesados en el campo.

Los estudios acerca de la relación entre psicología e historia han mostrado diversas maneras de interacción entre estas dos disciplinas. A este respecto, por ejemplo Gergen (1998) señala que cuando a una y a otra se les concibe como campos de saber independientes, se da origen a tres maneras de interactuar. En el primero, la psicología presenta sus objetos con un estatus "esencial". Es decir, estos objetos resultan inmutables en el tiempo, siempre han existido, solamente hacía falta que la investigación psicológica los descubriera como parte de una subjetividad considerada natural y por tanto universal. En este caso, en términos generales, entre la psicología y la historia se presenta un lazo que se restringe a ubicar el avance de la psicología como una serie de superaciones de errores. Un segundo camino da la vuelta a la relación y supone que la historia constituye lo psicológico. Como ilustración de este estilo de interacción, Gergen llama la atención sobre la cita de Vygotsky: "no hay nada en la mente que no haya estado primero en la sociedad" (p. 20). Es decir, en esta manera, el naturalismo queda desplazado y la cultura entra a jugar el papel central en la construcción de la historia de la disciplina. En este camino, la psicología se "torna en tributaria de la historia" (p. 21), en la medida en que los fenómenos psicológicos se convierten en producto de las condiciones históricas que los anteceden. Una tercera vía de acuerdo con el autor se preocupa por la génesis del discurso psicológico con el propósito de encontrar el lugar que tiene y las operaciones que este cumple en las sociedades contemporáneas.

Sobresale una característica en los trabajos aquí ubicados. Estos buscan hacer una crítica moral a 
la psicología, razón por la cual, se apartan de exclusivamente reflexionar sobre la naturaleza del pasado. Su objetivo, entonces, se relaciona con la alteración de las formas de acción cultural a través de la historización de la psicología y de su papel en referencia a la subjetividad psicologizada.

En esta misma línea que considera la relación entre psicología e historia, Rose (1996) después discutir las diferentes formas en las cuales ha sido construida la historia de la disciplina en referencia a aspectos económicos, sociales, culturales, de género, profesionales y políticos, se apoya en los trabajos de los investigadores en el campo de los estudios sociales de la ciencia, que han continuado las perspectivas y las herramientas propuestas en el trabajo de Michel Foucault (1998), para formular una propuesta que la titula como historia critica de la psicología, las cual se aparta tanto de querer celebrar el triunfo de la diseminación y el mejoramiento continuo de la psicología, como de proponer que se necesita un nuevo tipo de conocimiento psicológico sobre el sujeto.

En vez de ubicarse fundamentalmente en el discurso como una cuestión básicamente de negociación de significaciones, tal como lo hace Gergen (1998) en su examen socio-contruccionista de las perspectivas psico-históricas anteriormente mencionadas, Rose (1996) señala que la psicología debe considerarse básicamente como una cuestión tecnológica. Con esta premisa, la historia de la psicología se convierte en un análisis de las condiciones en las que se hace posible la práctica psicológica. Se asume, como propuso Foucault (1998) que no es el discurso psicológico el que inicialmente hace una serie de preguntas y proporciona una serie de respuestas acerca de los seres humanos, más bien, este conocimiento y sus prácticas derivadas surgen para dar respuesta a una gama de interrogantes acerca de las dificultades y contradicciones que la cotidianidad involucraba en referencia a la administración de los seres humanos en ámbitos institucionales. Entiéndase aquí la práctica como una convergencia en donde "lo que es dicho y hecho, las reglas impuestas y las razones, lo planeado y lo dado por hecho se encuentran e interconectan para producir cierta clase de sujetos" (Foucault, 1991, p. 75).
Más específicamente en palabras de Rose (1996, p. 11) la psicología se trata de una tecnología humana que puede ser descrita como: "un conjunto de artes y destrezas que implica la vinculación de pensamientos afectos, fuerzas artefactos y técnicas que no solamente fabrican y manipulan al ser, sino que, fundamentalmente lo ordenan, lo enmarcan, lo producen (...)". La noción de "complejo psi" que emergió en la psiquiatría crítica ha sido traída a este modo particular de considerar el pasado del conocimiento psicológico (Hook, 2004; Ingleby, 1985; Parker, 2007; Rose, 1985). Esta noción ofrece puntos centrales para la investigación histórica. Por una parte desplaza al conocimiento psicológico de ser un saber básicamente de las ideas y lo reubica en la articulación entre elementos que lo han hecho, y lo hacen, posible. Entre estos se encuentran solamente por mencionar algunos: revistas, libros, películas, profesionales, universidades, agremiaciones, programas televisivos, consultores de diferentes tipos, gurús, estudiantes, instrumentos, cuestionarios, encuestas e intervenciones de autoayuda.

Por otra parte, este desplazamiento de las ideas a las prácticas implica también un cuestionamiento a la dicotomía establecida a lo largo de la historia de la psicología entre el saber académico y la psicología popular. Se pone entonces bajo examen el supuesto que prescribe la separación que hace científico a uno y perteneciente al sentido común a la otra. En términos de la psicología como "complejo psi”, es decir, como puesta en escena de los elementos que la hacen posible, no se presentarían rupturas absolutas entre estas dos, sino más bien cierta continuidad que da origen a un ciclo en el cual lo público y lo privado, lo subjetivo y lo social entran en interacción gracias a, y en razón de, la psicología (Mckinley, 2003; Parker, 2007; Pulido, 2006; Pulido, García, Carvajal, \& González, 2013; Rose, 1988; Ward, 2002).

En términos generales, los estudios acerca del desenvolvimiento del "complejo psi" muestran una red, en donde las conexiones, las asociaciones y las interacciones han hecho posible la constitución histórica de la subjetividad en términos psicológicos. Sin embargo, estas investigaciones muestran una carencia central, pues han dejado de lado tanto la 
El "Museo de la psicología” como estrategia para la

CONSTRUCCIÓN DE LA HISTORIA ORAL DE LA PSICOLOGÍA

experiencia de aquellos que han actuado en la cotidianidad en su calidad de agentes psicológicos como la vivencia de esos otros que se han encontrado en las posiciones de audiencia, o de receptores, de las prácticas psicológicas (Apodaka, 2011; Illouz, 2010; Murray-Li, 2007). Entonces, de cierta forma, los estudios sobre el "complejo psi" no han considerado lo más común y corriente de la psicología. Quiere esto decir que se ha puesto entre paréntesis o no se le ha dado suficiente atención en estas investigaciones, a la interacción cotidiana, seguramente por considerarla obvia, entre los psicólogos practicantes y su público. Vale la pena insistir en que aun en estas aproximaciones abarcadoras para examinar el pasado de la psicología en las cuales se unen elementos de muy diverso orden, y en donde se considera la empresa psicológica en su conjunto más allá de escuelas particulares, se ha hecho caso omiso de lo, ciertamente, más "mundano" de la psicología. Esta situación se permea incluso en trabajos que se acercan a la historia de la "psicología práctica" como aquellos adelantados por Jansz y Van Drunen (2003), en donde la experiencia diaria, tanto de los agentes psicológicos como de aquellos que son objeto de su saber, se ha dejado sin considerar.

Quizá este tipo de omisiones esté relacionado con la proscripción de la experiencia cotidiana hecha en la construcción de las historias documentales. Se objeta como argumento central en ese tipo de historias que los relatos de la experiencia no son lo suficientemente "precisos" y que, por tanto, no se pueden dar por ciertas las elaboraciones que realizan las personas de sus vivencias anteriores. Por el contrario, los investigadores preocupados por la recuperación de la experiencia señalan, por ejemplo, la complementariedad que resulta en las maneras de narrar el pasado que utilizan tanto las fuentes documentales como históricas. Tal como señala Portelli (2003), el trabajo histórico que emplea fuentes orales es inconcluso por la naturaleza de las fuentes; el trabajo histórico que excluye las fuentes orales (cuando están disponibles) es incompleto por definición. En esta tensión entre fuentes, se da cabida a otras maneras de ocuparse del pasado de la disciplina, utilizando como herramienta a las historias orales con las consiguientes posibilidades y limitaciones que esto representa, cuestiones que serán consideradas a continuación.

\section{Historia oral y psicología}

Para examinar las implicaciones provenientes de la utilización de la historia oral para la construcción de la historia de la psicología, hay necesariamente que señalar cómo dentro de la disciplina histórica ha sido considerado el lugar que tiene la experiencia referida por los participantes en un evento. A este respecto, bien se conoce que los relatos de la experiencia de aquellos involucrados en determinadas situaciones se les han asignado diferentes papeles. De marginalizárseles como datos sesgados, se ha pasado a considerárselos como una forma diferente de hacer historia. En esta jornada, la historia oral poco a poco se alejó del puesto que le fue asignado inicialmente como un estilo de historia subordinada por imprecisa. El camino recorrido por la historia oral puede especificarse aún más para mostrar las ventajas que representa así como las limitaciones que muestra.

Los investigadores señalan que dicha evolución comienza con una desconfianza marcada de los historiadores convencionales acerca de los relatos que se obtienen de los testigos directos (Thomson, 1998, 2007). Se alegaba que los relatos son vagos e inexactos, así como cambiantes con el tiempo. Se argüía que la memoria no permanece estable y que por tanto estas narraciones no pueden convertirse en elementos confiables para establecer lo que realmente pasó. En este sentido, señala Thomson que aquellos interesados en la recolección de historias orales se dieron a la tarea de hacer más confiable a la historia oral, recurriendo a una serie de aspectos metodológicos desarrollados en la sociología, la antropología y en la psicología social para poder estandarizar los relatos y hacer comparaciones entre ellos, de forma tal que se comportaran más acordes con la ortodoxia positivista. Quizá el cambio más significativo acerca del lugar de la historia oral se ubicó en los años setenta, cuando, según Thomson (2007), los investigadores les dieron un vuelco a las críticas que señalaban las debilidades de la historia oral. Se propuso entonces que en vez de ser estas 
debilidades una cuestión critica que la invalidaba, estas objeciones representaban precisamente ventajas para la investigación histórica. Entonces, a la falta de confiabilidad de la memoria se le atribuyó precisamente la fortaleza de la historia oral. La variabilidad de la memoria empezó a representar un medio de acceder al significado subjetivo de la experiencia histórica, por tanto se convirtió en un modo para entender la relación entre el presente y el pasado, no solamente al nivel individual sino también colectivo (Thomson, 1998, 2007).

De acuerdo con Thompson (2007), la historia oral se convirtió así en un vehículo para explorar aspectos antes no considerados, tales como, la naturaleza del proceso de memoria histórica, la maneras en las cuales la gente le da un sentido a su pasado, las conexiones entre experiencia individual y el contexto social, las formas en que el pasado se convierte en parte del presente, así como los usos del pasado para interpretar las vidas actuales de los protagonistas. Hacia los años noventa, la preocupación de los investigadores se desplazó hacia la consideración de su propio lugar en el proceso de investigación. Si bien tanto los documentos escritos como en las historias orales no se producen en el momento en que ocurren los hechos (Vidal, 1990), en la historia oral el investigador crea sus fuentes, contribuye en gran medida a la producción de los datos y realiza la interpretación. Luego, la reflexividad se introdujo como herramienta para alcanzar un tipo de objetividad particular dentro del proceso de construcción de las historias orales y de su interpretación (Thomson, 2007). Implica esta reflexividad considerar la discusión que se adelanta en el mundo académico, sin caer en una intelectualización de las narraciones particulares de los participantes, también implica tener presente que este tipo de investigación se desenvuelve en una relación directa entre la persona contando sus experiencias y aquella que las recibe en un medio lleno de cuestiones políticas, económicas y sociales.

Es claro, como han señalado Barbosa y Rebello de Souza (2009), que hacer historia oral envuelve más una manera de ver la investigación en historia de la psicología que simplemente asumir una metodología para realizarla. Por supuesto, extendiendo el argumento de Portelli (2003), en términos generales para la historia oral, cuando esta se utiliza para acercarse al pasado de la psicología, se está considerando menos la verdad de los acontecimientos ocurridos y más el significado que estos tienen. Es a través de la reelaboración de la propia experiencia, con y de ser parte de la disciplina, en donde las imperfecciones del recordar cobran sentido en referencia a las maneras particulares de narrar de cada persona. Y posiblemente, si el estudio es suficientemente amplio, estas particularidades también puedan cobrar significación para un colectivo que puede estar constituido por alguna característica, escogida por los investigadores, asunto que por demás liga a las personas con algún atributo, como por ejemplo ser parte de una generación de profesionales (Barbosa \& Rebello de Souza, 2009; Portelli, 2003).

Las ventajas de la historia oral para la construcción de la historia de la psicología empiezan a ser consideradas a partir de los resultados de estudios realizados en otras áreas. Al ser trasladadas estas ventajas en referencia a la construcción de una narración histórica de la disciplina psicológica, por ejemplo, se puede considerar que la historia oral puede usarse para actualizar las versiones de aquellos que no están considerados dentro de los cánones académicos como figuras de la disciplina. De este modo, se pueden conocer, desde la perspectiva de los propios psicólogos, aspectos de la práctica cotidiana asociados con los procesos de expansión, o de psicologización, de la sociedad que no se encuentran consignados en documentos escritos.

En el caso particular de la elaboración de la historia de la psicología, campo en el cual se ha establecido un cierto equilibrio en términos de su construcción entre aquellos historiadores profesionales y los psicólogos que se acercan a la historia de la disciplina (Brock, 1995; Danziger, 1994), la historia oral representa una oportunidad para producir relatos en donde, como señala Danziger, los psicólogos, en su calidad de "outsiders" siendo "insiders", tienen la posibilidad de reflexivamente observar su papel como psicólogos y como entrevistadores constructores de historia. Esta situación además ubica a los psicólogos historiadores en una 
El "Museo de la psicología” como estrategia para la

CONSTRUCCIÓN DE LA HISTORIA ORAL DE LA PSICOLOGÍA

cierta posición horizontal con aquellos a quienes entrevista. Son básicamente dos o más psicólogos interactuando en una situación de compartir la experiencia en la cual, como señala Portelli (2003) aparecen el conflicto y la confrontación debidos no solamente a posiciones académicas diferentes, sino también a posiciones políticas que no están ligadas únicamente a asuntos psicológicos. No obstante, también aparece la complementariedad en la medida en que ambos están embarcados en la empresa de reconstruir la historia de la psicología.

Quizá uno de las más importantes ventajas de la historia de la psicología como historia oral está representada en la ganancia que en términos subjetivos se adquiere de la relación con el pasado de la disciplina. De acuerdo con Portelli (2003), como los análisis que se realizan a través de la historia oral están más concentrados en los procesos de creación de significaciones, el esfuerzo por conservar el pasado se aleja de aspectos factuales. En su lugar, se hace énfasis en comprender cómo se le da sentido al pasado a través de la elaboración que constantemente se lleva a cabo a través de recordar. Portelli señala que esto no quiere decir que el pasado factual no importe, pues los narradores son capaces de hacer una mirada a lo que pasó y de reconstruir sus actitudes, posiciones, prácticas, así como de dar razones por las cuáles algo ocurrió. Entonces, historias orales tienen una validez factual, pues suelen, solamente como ilustración, revelar acontecimientos desconocidos y también ubicar aspectos excluidos de acontecimientos bien conocidos (Portelli 2003). En aspectos relacionados específicamente con la construcción de la historia de la psicología, según Barbosa y Rebello de Souza (2009) se pueden establecer posiciones divergentes de las personas con respecto a ciertos hechos, se logra penetrar en aspectos que ningún otro método para construir la historia puede hacer y se contribuye a corregir los registros escritos que se han tomado por acabados. Todo esto se produce en la medida en que se dé el espacio para que en la relación entre investigador e investigado discurra, a través de la narración, lo exterior al individuo y la intimidad de este, el presente y el pasado (Barbosa y Rebello de Souza, 2009; Thompson, 2004).
En términos generales, la historia oral proporciona una mirada desde los propios protagonistas a la puesta en escena de la psicología en una perspectiva temporal. Esta versión histórica del "complejo psi” señala cómo este actúa en la relación entre lo íntimo y lo público, al nivel de la narración actualizada de las vivencias pasadas. Se produce un resultado fundamental en este proceso. En la medida en que se trabaja desde la elaboración que hace la memoria del pasado del "complejo psi", se dan pistas acerca de los caminos que puede tomar la disciplina, y como consecuencia, en razón a que la psicología al considerar al sujeto contribuye a producirlo, se empiezan a indicar formas que podría asumir la subjetividad y la cultura psicológica futuras (para esta discusión ver Castel et al., 1980; De Vos, 2013).

\section{El museo como estrategia metodológica para la producción de la historia oral}

Si bien en la literatura se presentan algunas recomendaciones acerca de cómo se debe hacer la historia oral, no existe una única serie de prescripciones para realizarla. Algunos recomiendan proponer un tópico general y desde ahí dejar discurrir libremente a los entrevistados; otros, por el contrario, señalan la importancia de hacer un diseño muy específico que responda con claridad a unas preguntas de investigación (Perks y Thomson, 2003). Efectivamente, en gran medida, el modo de adelantar la recolección de la información así como su interpretación a la luz de un marco conceptual específico, depende del investigador. En esta línea, y aunque no se quisiera, se mantiene, aunque atenuada, una disimetría en términos de los temas, las preguntas, los ritmos, los tiempos de investigación. Esta cuestión se ha tratado de minimizar, por parte de los investigadores, utilizando como táctica un mayor involucramiento de los participantes, por ejemplo, en la interpretación de sus propias narraciones.

En este sentido, se diseñó la estrategia para la construcción de un "museo de la psicología", eje fundamental de este artículo. Se buscaba disminuir la mencionada asimetría; apartarse de las maneras convencionales de recoger las narraciones y promover la confección de la historia oral de la psicología 
con un foco en la experiencia de la práctica que han desempeñado los psicólogos, con el objetivo de contribuir a llenar un vacío en el conocimiento. Metodológicamente, la estrategia se inspiró en la novela "El museo de la inocencia" de Orhan Pamuk (2011). La idea central consiste en que los investigadores proporcionen una serie de objetos, para que los psicólogos participantes puedan escoger aquellos que consideren dignos de hacer parte de un "museo de la psicología" y para que, una vez escogidos dichos objetos, se puedan narrar las razones por las cuales deben ser incluidos como parte del museo.

La selección de los elementos a disposición de los participantes para la construcción de "su" museo se hizo con distintos criterios y a través de diferentes fases. En primer lugar, se tuvo en cuenta la población que iba a participar. Este "museo de la psicología", iba a ser erigido para suscitar la narración de un grupo de psicólogos que se reunían con motivo de celebrar los cincuenta años de la Facultad de Psicología de la Pontificia Universidad Javeriana. Para tal efecto, estaban invitados, en distintas fechas, los egresados de cada una de las décadas desde que fue fundada la Facultad. Esta invitación a reencontrarse se constituía en ambiente propicio para el recuerdo, y a la vez en oportunidad para que en lo cotidiano, lejos de un contexto convencional de investigación, se recuperaran versiones de lo que había sido la práctica psicológica para la formación de los psicólogos, adelantada en diferentes décadas. La construcción del "museo de la psicología", considerada en este artículo, se llevó a cabo con los psicólogos egresados durante la década de los años 80 .

Una vez establecido quienes serían los participantes, se procedió a realizar una investigación de archivo en donde se examinaron los documentos concernientes a la situación de la psicología en la década bajo estudio. Se consultaron entonces los currículos, los libros y manuales utilizados, las descripciones contenidas en fichas de las prácticas académicas de la época, las publicaciones realizadas por la Facultad, los folletos de los eventos académicos, las imágenes de la infraestructura de la Facultad y de la Universidad. Con esta revisión se configuró una primera lista de objetos. En un segundo momento, se entrevistaron psicólogos que egresaron en esos años para que sugirieran objetos relacionados con la práctica psicológica durante el periodo escogido. De allí, se pudo establecer que adicionalmente a los elementos "puramente" psicológicos, se debería incluir objetos vinculados con los eventos sociales, económicos, políticos, tecnológicos y culturales que tenían conexiones con la práctica psicológica y que mostraban dimensiones del "complejo psi", constituido en referencia a lo que ocurrió en la época.

Se conformó entonces una lista que incluyó, por ejemplo, reseñas de los eventos sociales, fotografías de las tragedias naturales en los que se vieron involucrados los psicólogos y los estudiantes, los afiches de las abundantes películas "psicológicas" de los ochenta, máquinas de escribir, esténciles, papel carbón, "liquid paper", juguetes comúnmente usados en los consultorios, batas de laboratorio, diplomas, formularios de la biblioteca. Fotografías de profesores y de otras figuras que marcaron la época fueron también puestas a disposición. Ante la carencia de objetos "reales" que pudieran evocar el relato, se incluyeron imágenes impresas de situaciones ligadas a la psicología.

En un tercer momento, fue visitado el laboratorio de psicología, para ubicar allí diferentes clases de instrumentos psicológicos, baterías y hojas de respuestas (por ejemplo, láminas del test de Blacky y del test de Rorschach, así como los manuales y protocolos de respuesta), historia clínicas anónimas, lámparas de interrogatorio, un polígrafo, esqueletos plásticos y ejemplares artificiales de cerebros, que eran corrientemente utilizados. En total se contó con 120 objetos que se distribuyeron en diferentes mesas alrededor del salón en donde se reunieron los psicólogos. Estos objetos no guardaron una jerarquía entre sí. Solamente se constituyeron tanto en vehículos para evocar las vivencias significativas del pasado como en factores catalizadores de las emociones asociadas con dichas experiencias. Finalmente, hay que señalar que para complementar el ambiente apropiado para evocar el recuerdo se consideró pertinente que, simultáneamente, se proyectaran en el salón, imágenes de la universidad, de la ciudad y de la malla curricular del programa de psicología durante la década bajo examen. 
El "MUSEO de LA PSicología” COMO ESTRATEgia PARA LA

CONSTRUCCIÓN DE LA HISTORIA ORAL DE LA PSICOLOGÍA

Para iniciar la construcción del "museo de la psicología", se les aclaró a los asistentes que eran ellos quienes iban a seleccionar los objetos que formarían parte de este museo. Después de que los participantes habían seleccionado sus objetos, miembros del equipo de investigación procedieron a formular las siguientes preguntas a cada una de las personas: ¿qué escogió?, ipor qué?, iqué significado tiene ese objeto para usted?, icuál cree usted que es un objeto que falta en este museo?, iqué objeto debería excluirse de este museo? La información resultado de estas preguntas fue consignada en video para su posterior transcripción y análisis.

\section{De lo ocurrido en la construcción del "museo de la psicología"}

En términos generales, la puesta en escena de la psicología a través de la historia oral mediada por los objetos no ha sido suficientemente considerada. Por tanto, la experiencia que se presenta en este artículo, de cierta forma, fue pionera. Hace relativamente poco los investigadores se han empezado a preocupar por la materialidad en medio de la cual se ha hecho posible la práctica psicológica a lo largo de la historia. Esta perspectiva significa un apartarse, al menos en un primer momento, de la relaciones entre las personas, para privilegiar el lazo que se establece entre estas y los objetos. Se ha señalado como, aun en los enfoques más críticos de la historia de la psicología, las características concretas del medio objetual en el que se adelanta la práctica psicológica no ha llamado la atención de los investigadores. Por ejemplo, se ha descrito como el consultorio en donde Freud atendía estaba lleno de diversos tipos de elementos que despertaban la curiosidad de los pacientes, quienes en determinando momento "asociaban libremente" su discurrir con alguno de ellos, sin embargo, no ha sido objeto de mayor estudio este escenario montado para llevar a cabo el psicoanálisis (Mayer, 2006).

Cuando se considera la puesta en escena de la psicología en el tiempo, por supuesto los objetos que hacen parte de ella se tornan centrales. Al igual que los objetos, en la institución museo, en la estrategia que se propone, son de cierta manera sacados de su contexto social, para que el que se los apropie los tome para sí al adjudicarles contenidos elaborados por la memoria. Se trata entonces de un espacio lúdico y ritual a la vez (Navajas, 2007), en donde alrededor del objeto se conecta lo íntimo de la persona y lo público, asociado a lo material, y de paso se hace emerger en la narración la emocionalidad actual ligada a una situación pretérita. Como contemporáneamente se está discutiendo en la literatura acerca de las ventajas de la investigación cualitativa, en la construcción del "museo de la psicología" emergió, como punto central, la emocionalidad vinculada a los objetos, aspecto esencial que está prácticamente confinado al destierro en otros tipos de investigación relacionada con el pasado (Pulido \& Carvajal, 2010; Reilly, 2010).

A manera de ilustración del funcionamiento del "museo de la psicología" para la construcción de una historia oral de la disciplina, se presenta el siguiente fragmento obtenido durante la aplicación de la estrategia.

E. —iQué escogió?

P. - Yo escogí este libro, es un libro que publicó Henríquez, con las ponencias en un simposio que se llamó iEs posible una psicología molecular? Creo que fue en el 80 pero ya te confirmo, dice aquí en el 84 , (afirmando) en el 84. Para ese entonces, nosotros Olga Lucia y yo teníamos un sueño respecto de la unificación de la psicología, nos sentíamos incómodos en esto de que el hombre es una unidad y la psicología estuviera tan fraccionada en escuelas, en enfoques, en corrientes. Queríamos proponer la unificación de la psicología, como en efecto lo hicimos, nuestro trabajo de grado fue esa propuesta, en un principio era ideal, fue una quijotada cuando supimos que ese libro se había publicado y que se había realizado ese simposio nosotros no nos sentimos solos, sentimos que había mucha más gente y gente muy autorizada detrás de esa idea y fue un espaldarazo muy grande para nosotros, y fue un libro que leímos con pasión, con fruición con detalle y que nos sirvió muchísimo para comenzar nuestro trabajo y porque creemos que es un sueño en el cual todavía se puede pensar, porque nosotros aun soñamos el día en que la psicología sea una, el saber sea uno como el ser humano es uno. 
Pueden verse en este fragmento varios aspectos de la recuperación del pasado suscitados a través del libro escogido para hacer parte del "museo de la psicología". El objeto libro como tal deriva en la narración por dos caminos. Por una parte, resulta en un objeto que concentra conocimiento de una época y, por otra, focaliza la significación atribuida y la apropiación hecha de los contenidos de este, en la cotidianidad de la formación de estos psicólogos.

En el primero, escoger un libro que se publicó hace 30 años, del cual difícilmente se da cuenta contemporáneamente en la formación, señala el hecho factual de la forma que tomó en ese momento una de las discusiones centrales de la disciplina, e indica la importancia de este debate para la práctica psicológica del narrador en su paso por la vida universitaria como estudiante. Para ubicar el recuerdo, el narrador acude a comprobar su veracidad recurriendo de inmediato al objeto mismo para situarlo en la fecha de edición como evidencia de que lo acaecido en los años en que fue editado. Por supuesto, el libro como texto escrito ya hacía referencia, sin necesidad de utilizar relatos orales adicionales, a la centralidad que tuvo esta discusión durante la década. El libro es uno de los elementos que estaba relacionado con el interés del narrador, pues bien se señala en el fragmento que el participante estaba bastante involucrado en el debate. El objeto libro entonces se ha convertido principalmente en un desencadenador de la narración de la experiencia, puesto que bien pronto esta se desprende de los asuntos factuales para fluir del libro hacia la experiencia de la práctica psicológica. Es decir, la narración se aparta del libro en sí para dirigirse hacia su apropiación, permitiendo la elaboración presente de la experiencia pasada a través de la narración. La significación que asume el objeto libro se hace explícita y completa cuando se la vincula con el ejercicio de hacer un estudio. En la historia que se relata, esta discusión resulta tan importante para el narrador, y seguramente para aquellos que con él resuelven hacer la tesis sobre este tema, que toma la decisión de considerar el problema epistemológico para darle una respuesta.

Emerge entonces uno de los aspectos más importantes al que se tiene acceso gracias a la historia oral: la emocionalidad vinculada con la situación que el objeto desencadenó. Los asuntos racionales dan paso a los afectos que se asocian con el pasado. Un sueño, la incomodidad, la quijotada, la soledad, el espaldarazo, la pasión, la fruición son palabras atadas a sentimientos acerca de lo que para el narrador significó como hacer una tesis. El fragmento da cuenta de una empresa epopéyica cimentada a través de este "trabajo de grado" que tenía como objetivo transformar el conocimiento psicológico. La racionalidad asociada a ciertas formas de ver el avance de las disciplinas no tiene lugar, por el contrario, se señala cómo a través de una emocionalidad vinculada a unas condiciones históricas, se hace posible que las construcciones conceptuales se apropien, con el objetivo de transformar la disciplina misma.

Los sentimientos que aparecen hablan de las maneras de ser estudiante de psicología en los años ochenta, en una facultad de una universidad Jesuita. La fuerza del relato mismo difícilmente podría superarse por un análisis de textos previamente escritos a este respecto. Aun cuando las palabras están mediando la emocionalidad, con las limitaciones que esto tiene para transmitir la intensidad de los sentimientos, difícilmente puede superarse con datos provenientes de textos institucionales. El significado que le imprime el narrador a su vivencia para transmitirla a otros, pasa por las emociones vinculadas, por esta razón, el fragmento compele, se torna empático, fácilmente identificable y por tanto creíble. Esta relación con el pasado que se permea en el relato es uno de los aspectos más valiosos que aporta la "historia viva" y "desde abajo", calificativos comúnmente usados para denominar a las formas de construir relatos históricos basados en experiencias particulares.

Ahora bien, en este análisis también hay que resaltar que en el fragmento se unen sentimientos y agencia, dentro un contexto de formación de psicólogos en la década en cuestión. Se infiere que en la formación los contenidos epistemológicos eran parte importante de este proceso, aspecto que se confirma cuando se examinan los currículos de la época que se conservan en los archivos de la facultad. En referencia a currículos aparecen los espacios formativos que se ofrecían a los estudiantes de, muy 
El "Museo de la psicología” como estrategia para la

CONSTRUCCIÓN DE LA HISTORIA ORAL DE LA PSICOLOGÍA

importante notarlo, pregrado para que estuvieran en capacidad de decidir, proponer y realizar un estudio que se planteara preguntas muy complejas al punto de estar relacionadas con aquellos problemas que han atravesado la disciplina desde sus comienzos. No solamente señala la situación de una facultad, sino también condiciones del aparato educativo referido a la educación superior en el país, por ejemplo, para que este pudiera ofrecer profesores que guiaran a estudiantes de pregrado en una investigación compleja de tal magnitud.

Hay que hacer énfasis en que aquí se trabajó con un solo fragmento recogido a partir de la selección de objetos para construcción del "museo de la psicología", con el propósito de brevemente ejemplificar algunas posibilidades y limitaciones que ofrece la historia oral que de allí emerge. En el proceso con los psicólogos, cada uno de los objetos que compusieron el "museo de la psicología" suscitó narraciones diferentes. Es suficiente con indicar, por ejemplo, que a las fotografías de personajes de la época y de los profesores que estuvieron impartiendo las clases se les asoció una serie de prácticas para la formación, así como de rutinas personales que fueron conformando maneras de concebir el lugar de la psicología y de apropiarse de los contenidos que se les ofrecían por esa época a los estudiantes. Esta información con mucha dificultad se encuentra en archivos. Mejor aún, no se puede encontrar en los archivos en la medida en que las personas narran diversas versiones sobre el mismo objeto escogido, dando lugar al análisis precisamente de las variaciones que muestran formas de relación diferentes con los objetos como instrumentos para la construcción activa de significaciones sobre lo ocurrido. No se queda esta construcción del pasado en la indagación por lo acaecido y en la construcción activa del pasado. Se debe anotar de nuevo que la historia oral de la psicología sirve menos para establecer cuestiones factuales que para abrir nuevas preguntas de corte histórico.

\section{A manera de cierre}

Está claro cómo con la historia oral se adquiere un acceso al pasado "mundanal" de la psicología, des- de la perspectiva de los propios protagonistas. Las narraciones que se obtuvieron, de las cuales el fragmento solo es una muestra ilustrativa, manifiestan las vicisitudes que en la práctica tiene la psicología cuando lidia con la cotidianidad. Por supuesto, la historia oral es un medio para acceder al detalle de la psicologización, aquí, en términos del vínculo que se establece entre el pasado y el presente a través de la reelaboración de la vivencia de la formación de los protagonistas. Son estos quienes han tenido en su haber el hacer llegar la psicología a los rincones de la sociedad, para esto han tenido que formarse dentro en los cánones de la disciplina, esta empresa, con las diferentes dimensiones que implica como tal, difícilmente se conserva en registros de los archivos documentales en Colombia.

En este artículo, solamente se señalaron algunas de las posibilidades y limitaciones que tiene la construcción del "museo de la psicología", para la recolección de historias orales con miras a la construcción de una historia de la psicología. La estrategia representa una serie de ventajas a la hora de elicitar la narración. Es el participante quien escoge libremente entre los múltiples objetos de los que dispone, así como también puede hacer el reclamo por algún objeto que no está incluido. De este modo, quienes participan en el museo tienen mayor control sobre la narración, tanto en términos del tópico en general al cual se referirán como de las maneras en que lo harán. Se constituye una forma de investigación sobre el pasado que permite relaciones más horizontales entre el investigador y el investigado. A esta disminución de la disimetría también contribuye que ambos polos de la relación están constituidos por psicólogos, por tanto, se encuentran en una situación de poder relativo similar para controlar el relato y para discurrir sobre aspectos comunes provenientes de entrenamientos parecidos dentro de la disciplina.

Ya han sido consideradas en la literatura las ventajas que tiene la investigación cualitativa, por su facultad de suscitar y recoger la emocionalidad vinculada con los eventos sociales. Este aspecto es parte fundamental, en la medida en que estas narraciones reflejan directamente la experiencia de los investigados, para hacer más creíbles, cohe- 


\section{Hernán Camilo Pulido Martínez, luz Mery Carvajal Marín,}

Lina María Plata Castillo, Verónica Rivera Serna

rentes y significativas las construcciones propuestas, destinadas a diferentes tipos de audiencias. Al igual que para otras formas de hacer investigación de carácter cualitativo en donde los participantes reelaboran sus experiencias a través de algun tipo de mediación adicional (Reilly 2010; Cho \& Trent, 2006), en el "museo de la psicología", los objetos intermediarios permiten añadir fácilmente la dimensión emotiva que hace relatos más enriquecidos y potentes acerca del lugar ocupado por lo íntimo y lo público, en la relación presente y pasado en la historia de la psicología.

\section{Referencias}

Apodaka, E. (2011). La autoayuda y el consumo de dispositivos psíquicos en el caso de la innovación. ARBOR, Ciencia, Pensamiento y Cultura, 187(752), 1103-1118.

Barbosa, D. B., \& Rebello de Souza, M. P. (2009). Historia de psicologia: contribuições da etnografía e da história oral. Temas em Psicologia, 17(1), 81-91.

Brock, A. (1995). Why I am not a historian of science. History and Philosophy of Psychology Bulletin, 7, 27-31.

Castel, F., Castel, R., \& Lovell, A. (1980). La sociedad psiquiátrica avanzada. Barcelona: Anagrama.

Cho, J., \& Trent, A. (2006). Validity in qualitative research revisited. Qualitative Research, 6(3), 319340.

Danziger, K. (1994). Does the history of psychology have a future? Theory and Psychology, 4 (4), 467-484.

Danziger, K. (2001). Tres desafíos para la historia de la psicología. Buenos Aires: Departamento de Publicaciones/Facultad de Psicología. Disponible en www.elseminario.com.ar

De Vos, J. (2012). Psychologization in times of globalization. New York: Routledge.

Eghigian, G., Killen, A., \& Luenberger, C. (Eds.). (2007). The self as a project. Politics and the human sciences in the Twentieth Century (Serie Osiris, Vol. 22, pp. 1-25). Chicago: University of Chicago Press.

Foucault, M. (1979). Governmentality. IECC. Ideology and Conciousness, 6, 5-21.

Foucault, M. (1998). La psicología de 1850 a 1950. Revista Archipiélago, 34/35, 163-173.
Fraser, R. (1993). Historia oral, historia social. Historia Social, 17, 131-139.

Gergen, K. J. (1998). History and psychology: Three weddings and a future. En P. N. Stearns \& J. Lewis (Eds.), An emotional history of United States (pp. $15-$ 32). New York: New York University Press.

Gl】veanu, V., \& Yamamoto, K. (2012). Bridging history and social psychology: What, how and why. Integrative Psychological Behavior, 46(4), 431-439.

Gillespie, R. (1993). Manufacturing knowledge a history of the Hawthorne studies. New York. Cambridge University Press.

Herman, E. (1995). The romance of American psychology: Political culture in the age of experts. Berkely: University of California Press.

Hook, D. (2004) Critical psychology. Lansdowne: UCT Press.

Illouz, E. (2010). La salvación del alma moderna. Terapia, emociones y la cultura de la autoayuda. Madrid: Kazt.

Ingleby, D. (1985). Professionals as socializers: The 'psy complex'. Research in Law Deviance and Social Control, 7, 79-109.

Jansz, J., \& van Druner, P. (2003). A social history of psychology. Oxford: Blackwell.

Jaraba-Barrios, B., \& Mora-Gamez, F. (2010). Reconstruyendo el objeto de la crítica: sobre las posibles confluencias entre psicología crítica y estudios sociales de la ciencia. Revista Colombiana de Psicología, 19(2), 225-239.

Klappenbach, H. (2006). Construcción de tradiciones historiográficas en psicología y psicoanálisis. Psicologia em Estudo, 11(1), 3-17.

Lovett, B. J. (2006). The new history of psychology: A review and critique. History of Psychology, 9(1), 17-37.

Mayer, A. (2006). Lost objects: From the laboratories of hypnosis to the psychoanalytic setting. Science in Context, 19(1), 37-64.

McKinley Runyan, W. (2003). From the study of lives an psychohistory to historicizing psychology: A conceptual journey. The Annual Review of Psychoanalysis, 31, 119-134.

Murray-Li, T. (2007). The will to improve: Governmentality, development, and the practice of politics. Durham: Duke University Press. 


\section{El "MUSEO DE LA PSiCOlogía" COMO ESTRATEgIA PARA LA CONSTRUCCIÓN DE LA HISTORIA ORAL DE LA PSICOLOGÍA}

Navajas, O. (2007). Interpretar el museo. Boletín de Interpretación, 17, 7-8.

Pamuk, O. (2011). El museo de la inocencia. Barcelona: Mondadori.

Parker, I. (2007). Revolution in psychology: Alienation to emancipation. London: Pluto Press.

Perks, R., \& Thomson, A. (2003). The oral history reader. Nueva York: Routledge.

Pfister, J., \& Schnog, N. (1997). Inventing the psychological. Toward a cultural history of emotional life in America. New Haven: Yale University Press.

Portelli, A. (2003). What makes oral history different? En R. Perks \& A. Thomson (Eds.), The oral history reader (pp. 63-74). New York: Routledge.

Pulido, H. C. (2006). Produciendo trabajadores modernos: conocimiento psicológico y el mundo del trabajo en el sur. Universitas Psychologica, 6(1), 27-37.

Pulido, H. C., \& Carvajal, L. M. (2010). Construyendo un sentido en común dentro del área de gestión de recursos humanos de un hospital universitario. Universitas Psychologica, 10(2), 595-607.

Pulido, H. C., García, C. M., Carvajal, L. M., \& González, I. D. (2013). Entre bendiciones y prejuicios: mapeando el lugar y las operaciones de la psicología en relación con el trabajo en el sector de la salud en Bogotá. En O. Restrepo (Ed.), Ensamblado en Colombia (pp. 355-370). Bogotá: Colciencias.

Reilly, R. C. (2010, abril). Found poems as a dimension of representational trustworthiness. Ponencia presentada en la Qualitative Research in Management and Organization Conference, Albuquerque, Nuevo México, USA.
Rose, N. (1985). The psychological complex: Psychology politics and society in England 1869-1939. Londres: Routledge and Kegan Paul.

Rose, N. (1988). Calculable minds and manageable individuals. History of the Human Sciences, 1(2), 179-200.

Rose, N. (1996). Inventing ourselves. Psychology, power and regulation. Londres: Sage.

Rose, N. (1999). Governing the soul. The shaping of the private self. Londres: Free Association Books.

Samelson, F. (1999). Assessing research in the history of psychology: Past, present, and future. Journal of the History of the Behavioral Sciences, 35(3), 247-255.

Thomson, A. (1998). Fifty years on: An international perspective on oral history. The Journal of American History, 85(2), 581-595.

Thomson, A. (2007). Four paradigm transformation in oral history. Oral History Review, 34(1), 49-70.

Thompson, P. (2004). Historia oral y contemporaneidad. Historia, Memoria y Pasado Reciente, 20, 15-33.

Triantafillou, P., \& Moreira, A. (2005). Modern templates of happiness: Performing psychotechnics and spiritualism in Denmark. History of the Human Sciences, 18(2), 87-109.

Vezzetti, H. (1996). Los estudios históricos de la psicología en la Argentina. Cuadernos Argentinos de Historia de la Psicología, 2(1-2). Disponible en http://arielviguera.googlepages.com/Vezzetti_Estudios Historicos.pdf

Vidal, D. G. (1990). De Herodoto ao gravador: historias da historia oral. Resgate, 1, 77-82.

Ward, S. C. (2002). Modernizing the mind. Psychological knowledge and the remaking of society. Westport: Praeger Publishers. 
